\title{
Número de Grundy impróprio de subclasses de cografos *
}

\author{
Efraim Rodrigues ${ }^{1}$, Cláudia Linhares Sales ${ }^{1}$ \\ ${ }^{1}$ ParGO e Departamento de Ciência da Computação, Universidade Federal do Ceará, Brasil \\ efraimnaassom@gmail.com, linhares@lia.ufc.br
}

\begin{abstract}
The d-improper vertex coloring problem, where a color assigned to a vertex can be shared with at most $d, d \geq 0$, of its neighbors, is a generalization of the classic vertex coloring problem ( set $d=0)$. Being equally intractable, the greedy d-improprer coloring heuristic was introduced in [Rodrigues 2020]. In this work, we study the worst performance of this heuristic on some subclasses of cographs.
\end{abstract}

Resumo. O problema de coloração d-imprópria de vértices, que permite que uma cor atribuída a um vértice possa ser compartilhada com até $d, d \geq 0$, de seus vizinhos, é uma generalização do problema clássico de coloração de vértices (onde $d=0$ ). Sendo igualmente intratável, a heurística de coloração gulosa d-imprópria foi introduzida em [Rodrigues 2020]. Neste trabalho, determinamos o seu pior desempenho em algumas subclasses de cografos.

\section{Introdução}

Dado um grafo $G=(V, E)$, uma coloração dos vértices é uma atribuição de rótulos (cores) aos vértices $V(G)$. Uma coloração é dita própria se vértices adjacentes recebem cores diferentes. O problema clássico de coloração consiste em, dado um grafo $G$, determinar o seu número cromático $\chi(G)$ que é o menor inteiro $k$ tal que $G$ admite uma coloração própria com $k$ cores. Em geral, o problema de determinar $\chi(G)$ é NP-difícil [Karp 1972]. Problemas de alocação de frequências são geralmente modelados como um problema de coloração, mas em alguns casos as antenas toleram compartilhar suas frequências com antenas vizinhas até um certo limite $d$. Esses casos podem ser modelados como problemas de coloração defeituosa ou d-imprópria de vértices onde um vértice pode compartilhar sua cor com no máximo $d$ vizinhos. O problema de determinar se um grafo $G$ admite uma coloração $d$-imprópria com $k$ cores é NP-completo [Cowen et al. 1997].

Dada uma ordem dos vértices, considerando os vizinhos já coloridos de um vértice $v$, dizemos que uma cor $c$ está disponível para $v$ se nenhum vizinho de $v$ está colorido com c. O Algoritmo Guloso de Coloração usa a seguinte estratégia para colorir propriamente os vértices de um grafo: seguindo uma ordem dos vértices, a menor cor disponível é atribuída para cada vértice nessa ordem. O número de Grundy $\Gamma(G)$ de um grafo é o maior número de cores usadas em uma coloração pelo Algoritmo Guloso de Coloração considerando todas as ordens possíveis de $V(G)$. Esse parâmetro foi introduzido no contexto de grafos direcionados e com aplicação à jogos em [Grundy 1939] e posteriormente relacionado ao problema de coloração de grafos por

\footnotetext{
${ }^{*}$ Essa pesquisa foi financiada pelo CNPq (Grants 304831/2017-4 e 425297/2016-0) e CAPES (Grant 88887.197437/2018-00)
} 
[Christen and Selkow 1979]. Em geral, o problema de determinar o número de Grundy é NP-completo [Goyal and Vishwanathan 1997].

Tendo em vista a dificuldade do problema, é natural a proposição de heurísticas para abordá-lo. Em [Rodrigues 2020], foi introduzido o Algoritmo Guloso de Coloração d-imprópria e, como feito habitualmente, um parâmetro para medir o seu pior caso, o número de Grundy $d$-impróprio $\Gamma^{d}(G)$ de um grafo $G$.

Neste trabalho, nós investigamos $\Gamma^{d}(G)$ em algumas subclasses de cografos.

\section{Algoritmo Guloso de Coloração $d$-imprópria}

De forma semelhante ao Algoritmo Guloso de Coloração, dada uma ordem dos vértices de um grafo, o Algoritmo Guloso de Coloração $d$-imprópria colore cada vértice com a menor cor disponível considerando os vértices já coloridos. A impropriedade de um vértice $v$ indica o número de vizinhos coloridos com a mesma cor de $v$ [Cowen et al. 1986]. Uma cor $i$ está disponível para um vértice $v$ se $v$ tem no máximo $d$ vizinhos coloridos com $i$ e nenhum deles tem impropriedade $d$. Dizemos que um vértice $v$ está saturado se $v$ tem impropriedade $d$, e que uma cor é saturada em um conjunto de vértices se pelo menos um vértice do conjunto colorido com a cor é saturado. O número de Grundy d-impróprio de um grafo $G$, denotado por $\Gamma^{d}(G)$, é o maior inteiro $k$ tal que o Algoritmo Guloso de Coloração $d$-imprópria retorna uma coloração $d$-imprópria com $k$ cores, considerando todas as ordenações possíveis de $V(G)$.

Lema 1. [Rodrigues 2020] Seja $G$ um grafo qualquer e $d \geq 0$, então $\Gamma^{d}(G) \leq\left\lceil\frac{|V(G)|}{d+1}\right\rceil$. Se Gé um grafo completo, esse limite é justo.

Demonstração. Suponha que, por absurdo, $\Gamma^{d}(G)>\left\lceil\frac{|V(G)|}{d+1}\right\rceil$. Seja $u$ um vértice colorido com a cor $\left\lceil\frac{|V(G)|}{d+1}\right\rceil+1$. A existência de $u$ implica que existe uma cor $i, i<\left\lceil\frac{|V(G)|}{d+1}\right\rceil+1$, atribuída a menos de $d+1$ vértices. Como $u$ não pôde receber a cor $i$ e não existem $d+1$ vértices com a cor $i$ que pudessem ser adjacentes a $u$, isso implica que existe $v$ colorido com $i, u v \in E(G)$, tal que $v$ possui $d$ vizinhos com a cor $i$. Ora, isso implica que a cor $i$ é atribuída a pelo menos $d+1$ vértices, uma contradição. No caso de grafos completos, cada conjunto de $d+1$ vértices satura uma cor e, portanto, esse limite é justo.

O Lema 1 apresenta um limite superior trivial para o número de Grundy $d$ impróprio e consequentemente para o número cromático $d$-impróprio de um grafo $G$.

\section{Número de Grundy $\boldsymbol{d}$-impróprio de cografos}

Seja $P_{4}$ um caminho induzido com quatro vértices. Dizemos que um grafo $G$ é um cografo se $G$ é livre de $P_{4}$ 's induzidos. O grafo $G$ obtido pela união disjunta $(\cup)$ de $G_{1}$ e $G_{2}$ tem $V(G)=V\left(G_{1}\right) \cup V\left(G_{2}\right)$ e $E(G)=E\left(G_{1}\right) \cup E\left(G_{2}\right)$. O grafo $G$ obtido pela união completa $(+)$ de $G_{1}$ e $G_{2}$ tem $V(G)=V\left(G_{1}\right) \cup V\left(G_{2}\right)$ e $E(G)=E\left(G_{1}\right) \cup E\left(G_{2}\right) \cup\{(u, v) \mid u \in$ $\left.G_{1}, v \in G_{2}\right\}$. [Corneil et al. 1981] mostraram que um cografo pode ser definido recursivamente como segue: o grafo trivial é um cografo; se $G_{1}$ e $G_{2}$ são cografos, então o grafo $G$ obtido a partir da união completa ou disjunta entre $G_{1}$ e $G_{2}$ é um cografo. O teorema que segue trata do número de Grundy $d$-impróprio de grafos desconexos.

Teorema 2. [Rodrigues 2020] Seja $G=G_{1} \cup G_{2}$. Então, $\Gamma^{d}(G)=$ $\max \left\{\Gamma^{d}\left(G_{1}\right), \Gamma^{d}\left(G_{2}\right)\right\}$. 
Demonstração. Provamos que $\Gamma^{d}(G) \geq \max \left\{\Gamma^{d}\left(G_{1}\right), \Gamma^{d}\left(G_{2}\right)\right\}$ e $\Gamma^{d}(G) \leq$ $\max \left\{\Gamma^{d}\left(G_{1}\right), \Gamma^{d}\left(G_{2}\right)\right\}$ e o resultado segue. Dada uma coloração $d$-imprópria gulosa de $G$ com $\Gamma^{d}(G)$ cores, a necessidade de uma cor $\max \left\{\Gamma^{d}\left(G_{1}\right), \Gamma^{d}\left(G_{2}\right)\right\}+1$ só seria justificada se novas arestas fossem adicionadas em $G_{1}$ ou em $G_{2}$ ou entre $G_{1}$ e $G_{2}$. Uma vez que a união disjunta não cria novas arestas, o limite superior está provado. Por outro lado, como a união das colorações gulosas de $G_{1}$ e $G_{2}$ com $\Gamma^{d}\left(G_{1}\right)$ e $\Gamma^{d}\left(G_{2}\right)$ cores, respectivamente, é uma coloração gulosa $d$-imprópria de $G$ com $\max \left\{\Gamma^{d}\left(G_{1}\right), \Gamma^{d}\left(G_{2}\right)\right\}$ cores, o limite inferior está provado.

A seguir, tratamos de classes de cografos conexos. O seguinte resultado é trivial, uma vez que o grafo obtido pela união completa de dois grafos completos é completo.

Teorema 3. Sejam $G_{1}$ e $G_{2}$ grafos completos e $G=G_{1}+G_{2}$ com $|V(G)|=n$. Então, $\Gamma^{d}(G)=\left\lceil\frac{n}{d+1}\right\rceil$.

O exame da união completa de dois grafos sem arestas $G_{1}$ e $G_{2}$ deve ser feito, entretanto, mais detalhadamente. Primeiramente, observe que o resultado da união completa, nesse caso, é um grafo bipartido completo $G=\left(V\left(G_{1}\right), V\left(G_{2}\right)\right)$. Considere, sem perda de generalidade, um vértice $v$ qualquer em $V\left(G_{1}\right)$. O vértice $v$ estará proibido de receber uma cor $i$ em duas situações. Caso tenha mais que $d$ vizinhos coloridos com a cor $i$ (e isso implica ter mais que $d$ vértices coloridos com a cor $i$ em $V\left(G_{2}\right)$ ) ou caso tenha em sua vizinhança um vértice saturado colorido com a cor $i$ (e isso implica ter $d$ vértices coloridos com a cor $i$ em $V\left(G_{1}\right)$ ). Assim, uma coloração que satura uma cor $c$ em ambas as partes da bipartição colore $d$ vértices com $c$ em $V\left(G_{1}\right)$ e em $V\left(G_{2}\right)$.

Teorema 4. Sejam $G_{1}$ e $G_{2}$ grafos sem arestas onde $\left|V\left(G_{1}\right)\right| \leq\left|V\left(G_{2}\right)\right| e G=G_{1}+G_{2}$. Então, para $d \geq 1$ e $t=\left\lceil\frac{\left|V\left(G_{1}\right)\right|}{d}\right\rceil, \Gamma^{d}(G)=t$ se $\left|V\left(G_{2}\right)\right| \leq d t e \Gamma^{d}(G)=t+1$ caso contrário.

Demonstração. Seja $H$ um subgrafo induzido de $G$, obtido pela união completa de $G_{1}$ com $\left|V\left(G_{1}\right)\right|$ vértices de $G_{2}$. Uma ordem que alterna $d$ vértices de $V\left(G_{1}\right)$ com $d$ vértices de $V\left(G_{2}\right)$ colore sucessivamente $2 d$ vértices do grafo para cada cor $i$ saturada. Isso impede que a cor $i$ seja usada no restante do grafo. Logo essa ordem utiliza $\left\lceil\frac{|V(H)|}{2 d}\right\rceil$, ou seja, $t$ cores para colorir $H$. Se $\left|V\left(G_{1}\right)\right|=\left|V\left(G_{2}\right)\right|$, então $\Gamma^{d}(G)=t$.

Se $\left|V\left(G_{1}\right)\right|<\left|V\left(G_{2}\right)\right|$, considere então uma coloração $d$-imprópria ótima de $H$ com $t$ cores. Observe que todos os vértices de $G_{1}$ estão coloridos. Se $\left|V\left(G_{2}\right)\right| \leq d t$, os vértices não coloridos de $G_{2}$ podem receber a cor $t$ e $\Gamma^{d}(G)=t$. Agora, considere o caso em que $\left|V\left(G_{2}\right)\right|>d t$. Observe que todos os vértices de $G_{1}$ coloridos de 1 a $t-1$ estão saturados e os coloridos com $t$ podem tornar-se saturados ao final da coloração de $G$. Além disso, todos os vértices de $G_{2}$ que receberam as cores 1 a $t-1$ estão saturados. Os $\left|V\left(G_{2}\right)\right|-d t$ vértices não coloridos de $G_{2}$ não podem ser coloridos com cor menor ou igual a $t$, pois isso violaria a impropriedade de vértices de $G_{1}$. Note que a cor $t+1$ não ocorre em $G_{1}$. Ademais, $G_{2}$ não possui arestas. Portanto, esses dois fatos indicam que a cor $t+1$ pode ser usada em todos os vértices ainda não coloridos de $G_{2}$. Isso encerra a prova.

Observe que se $G$ é um grafo isomorfo ao $K_{2}$, o caso de $G$ é tratado por ambos Teoremas 3 e 4 . Usamos o fato de que o parâmetro $\Gamma^{d}$ é monotônico com respeito a 
subgrafos, ou seja, $\Gamma^{d}(H) \leq \Gamma^{d}(G)$ para todo $H \subseteq G$ (visto que a inclusão de arestas só pode aumentar o número de Grundy $d$-impróprio), para extrair resultados para a união completa de quaisquer cografos, considerando o caso $d=1$. A seguir apresentamos um limite inferior de $\Gamma^{1}(G)$ para grafos $G$ obtidos a partir da união completa entre dois cografos.

Teorema 5. Sejam $G_{1}$ e $G_{2}$ cografos quaisquer onde $\left|V\left(G_{1}\right)\right| \leq\left|V\left(G_{2}\right)\right|$, e $G=G_{1}+G_{2}$. Então, $\Gamma^{1}(G)=\left|V\left(G_{1}\right)\right|$ se $\left|V\left(G_{1}\right)\right|=\left|V\left(G_{2}\right)\right| e \Gamma^{1}(G) \geq\left|V\left(G_{1}\right)\right|+1$ caso contrário.

Demonstração. O grafo $G$ possui como subgrafo gerador o cografo obtido pela união completa de dois grafos sem arestas. Como a inclusão de arestas só pode aumentar o número de Grundy 1-impróprio, temos que os valores estabelecidos no Teorema 4 constituem um limite inferior para $\Gamma^{d}(G)$. Logo, usando o Teorema 4 para $d=1$, obte$\operatorname{mos} \Gamma^{1}(G) \geq\left|V\left(G_{1}\right)\right|$ quando $\left|V\left(G_{1}\right)\right|=\left|V\left(G_{2}\right)\right|$ ou $\Gamma^{1}(G) \geq\left|V\left(G_{1}\right)\right|+1$ caso contrário. Ademais, usando Lema 1 , no caso de $\left|V\left(G_{1}\right)\right|=\left|V\left(G_{2}\right)\right|$, obtém-se que $\Gamma^{1}(G)=\left|V\left(G_{1}\right)\right|$. Isso conclui a prova do teorema.

Esse trabalho prosseguirá com o estudo do parâmetro em cografos em geral e nas suas superclasses, de grafos com poucos $P_{4}$ 's, que são definidas pela quantidade de $P_{4}$ 's admitidos a cada certo número fixo de vértices.

Os autores são gratos aos pareceristas pela leitura cuidadosa e valiosas sugestões. Como foi observado por um dos pareceristas, todos os resultados aqui apresentados aplicam-se a grafos que são obtidos por união completa ou união disjunta de dois grafos quaisquer (não apenas de cografos). Essa observação é verdadeira, a nossa escolha em trabalhar com cografos dá-se pelo desejo de obter uma estimativa recursiva do valor do parâmetro. Contudo, essa observação pertinente nos leva a almejar o estudo do comportamento do parâmetro com respeito a outras operações em grafos.

\section{Referências}

Christen, C. A. and Selkow, S. M. (1979). Some perfect coloring properties of graphs. Journal of Combinatorial Theory, Series B, 27(1):49 - 59.

Corneil, D., Lerchs, H., and Burlingham, L. (1981). Complement reducible graphs. Discrete Applied Mathematics, 3(3):163 - 174.

Cowen, L., Goddard, W., and Jesurum, C. E. (1997). Defective coloring revisited. Journal of Graph Theory, 24(3):205-219.

Cowen, L. J., Cowen, R. H., and Woodall, D. R. (1986). Defective colorings of graphs in surfaces: Partitions into subgraphs of bounded valency. Journal of Graph Theory, 10(2):187-195.

Goyal, N. and Vishwanathan, S. (1997). NP-completeness of undirected grundy numbering and related problems. Unpublished manuscript.

Grundy, P. M. (1939). Mathematics and games. Eureka, 2:6-9.

Karp, R. M. (1972). Reducibility among Combinatorial Problems, pages 85-103. Springer US, Boston, MA.

Rodrigues, E. (2020). Coloração k-imprópria gulosa. Dissertação de Mestrado, MDCC Universidade Federal do Ceará. 